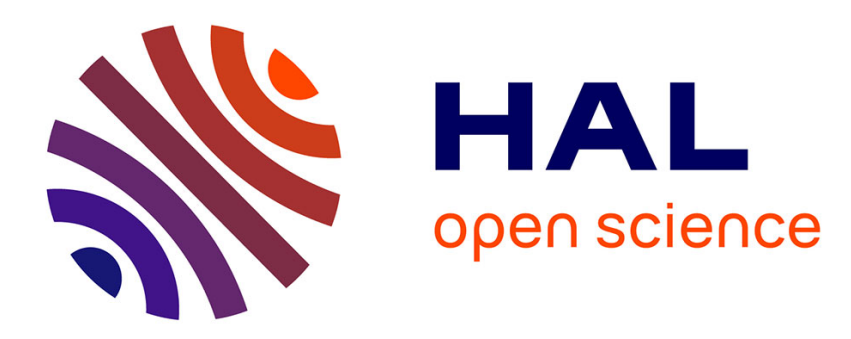

\title{
Etude de l'immobilisation de Lactobacillus helveticus dans des billes d'alginate de calcium
}

\author{
P. Boyaval, A. Lebrun, J. Goulet
}

\section{To cite this version:}

P. Boyaval, A. Lebrun, J. Goulet. Etude de l'immobilisation de Lactobacillus helveticus dans des billes d'alginate de calcium. Le Lait, 1985, 65 (649_650), pp.185-199. hal-00929046

\section{HAL Id: hal-00929046 https://hal.science/hal-00929046}

Submitted on 1 Jan 1985

HAL is a multi-disciplinary open access archive for the deposit and dissemination of scientific research documents, whether they are published or not. The documents may come from teaching and research institutions in France or abroad, or from public or private research centers.
L'archive ouverte pluridisciplinaire HAL, est destinée au dépôt et à la diffusion de documents scientifiques de niveau recherche, publiés ou non, émanant des établissements d'enseignement et de recherche français ou étrangers, des laboratoires publics ou privés. 


\title{
Etude de l'immobilisation de Lactobacillus helveticus dans des billes d'alginate de calcium
}

\author{
P. BOYAVAL*, A. LEBRUN et J. GOULET
}

\begin{abstract}
RESUME
Des cellules entières métabolisantes de Lactobacillus helveticus ont été immobilisées dans des billes d'alginate de calcium afin de fermenter en continu du perméat d'ultrafiltration de lactosérum. Les concentrations optimales de gélification ont été déterminées à $2 \%(\mathrm{M} / \mathrm{V})$ d'alginate et $3 \%(\mathrm{M} / \mathrm{V})$ de $\mathrm{CaCl}_{2}$ en fonction de la stabilité des billes vis-à-vis de la dissolution du gel, du relargage des cellules dans le milieu et des problème de diffusion des nutriments. Les billes présentent alors un diamètre moyen de $3,0 \pm 0,2 \mathrm{~mm}$. Le suivi du nombre de cellules viables intra-bille était effectué par la dissolution du gel dans un tampon citrate de sodium et dénombrement sur géloses MRS. Les traitements de stabilisation chimique du gel par l'emploi de glutaraldéhyde, polyéthylèneimine et de tannins se sont révélés trop toxiques pour les cellules ou inefficaces et n'ont donc pas été poursuivis. La conservation du biocatalyseur pour sa réutilisation a été envisagée à différentes températures : $-196^{\circ} \mathrm{C},-35^{\circ} \mathrm{C},-20^{\circ} \mathrm{C},+4^{\circ} \mathrm{C},+25^{\circ} \mathrm{C}$, dans du lait et dans du perméat de lactosérum. Les conservations dans l'azote liquide et à $+4^{\circ} \mathrm{C}$ étaient les plus efficaces. Les congélations lentes à $-20^{\circ} \mathrm{C}$ et $-35^{\circ} \mathrm{C}$ altéraient l'homogénéité de la bille et la survie cellulaire.
\end{abstract}

Mots clés : Lactobacillus helveticus - Immobilisation - Alginate - Perméat de lactosérum - Conservation.

Département de sciences et technologie des aliments, et Centre de recherche en nutrition, Université Laval, Québec, Canada G1K 7P4.

* Adresse actuelle : I.N.R.A., Laboratoire de recherches de technologie laitière, 65, rue de Saint-Brieuc - F 35042 Rennes cedex. 


\section{SUMMARY}

\section{IMMOBILIZATION OF LACTOBACILLUS HELVETICUS IN CALCIUM-ALGINATE BEADS}

Whole metabolizing Lactobacillus helveticus cells were immobilized in calcium alginate beads in order to ferment cheese whey ultrafiltrate. Optimal concentrations for gelification were determined as $2 \%(\mathrm{~W} / \mathrm{V})$ alginate and $3 \%(\mathrm{~W} / \mathrm{V}) \mathrm{CaCl}_{2}$, with respect to gel dissolution, cell leakage, and nutrient diffusion. The beads had an average diameter of $3.0 \pm 0.2 \mathrm{~mm}$. Cell viability was determined after complete dissolution of the beads in $1 \%(\mathrm{~W} / \mathrm{V})$ pH 6.0 $\mathrm{Na}^{+}$-citrate. Plate counts were done on MRS medium. Improvement of physical properties of gel beads with glutaraldehyde, polyethyleneimine and tannins as hardening agents were not satisfactory and no further employed. Storage of the beads were investigated at $-196^{\circ} \mathrm{C},-35^{\circ} \mathrm{C},-20^{\circ} \mathrm{C},+4^{\circ} \mathrm{C}$ and $+25^{\circ} \mathrm{C}$ in milk and in cheese whey permeate. Optimal conditions for preservation were $-196^{\circ} \mathrm{C}$ and $+4^{\circ} \mathrm{C}$. Slow freezing impaired cell viability and beads homogeneity.

Key words : Lactobacillus helveticus - Immobilization - Alginate - Cheese whey permeate - Storage.

\section{INTRODUCTION}

L'immobilisation de cellules entières n'est pas un nouveau concept mais l'exploitation à des fins analytiques ou industrielles d'un phénomène observé dans la nature; les avantages de l'emploi de cellules immobilisées sont nombreux (Durand et Navarro, 1978; Fukui et Tanaka, 1982). Parmi ceux-ci, l'on peut citer la séparation aisée du produit du catalyseur, la possibilité de régénérer le biocatalyseur pour un nouvel emploi, la moins grande susceptibilité de celui-ci aux contaminations. Si l'on compare les cellules immobilisées aux enzymes immobilisées qui, depuis les travaux de Nelson et Griffin sur l'invertase adsorbée sur charbon, se sont beaucoup développées, d'autres avantages apparaissent : il n'est pas nécessaire de purifier l'enzyme ou les enzymes avant l'immobilisation; de plus, le problème de la régénération des coenzymes lors des réactions est résolu par l'emploi de cellules entières viables, ainsi que celui de la stabilité des enzymes intracellulaires purifiées (Chibata et Tosa, 1981). Mais l'avantage primordial présenté par l'emploi de cellules entières réside dans la possibilité d'exploitation des voies multi-enzymatiques. Par exemple, la biosynthèse de coenzyme A à partir d'acide pantothénique, de cystéine et d'ATP 
implique cinq réactions consécutives. Shimizu et al. $(1975,1979)$ ont immobilisé des cellules de Brevibacterium ammoniagenes, grand producteur de ce coenzyme, par cinq méthodes différentes. Les préparations cellulaires immobilisées dans un gel de polyacrylamide ou confinées dans un tube de cellophane présentaient l'activité recherchée.

La production d'acide lactique à l'aide de cellules immobilisées semble une solution intéressante pour employer le perméat de lactosérum qui demeure un sous-produit encombrant de l'industrie laitière. Les procédés actuels de production d'acide lactique par cellules immobilisées se divisent en deux classes : l'utilisation de fibres d'ultrafiltration comme moyen de confinement et de séparation du produit des cellules et l'emploi de cellules immobilisées dans des billes de gel. Nous décrivons ici l'approche de la deuxième méthode dans laquelle des cellules entières de Lactobacillus helveticus sont immobilisées dans un gel d'alginate de calcium.

\section{MATERIEL ET METHODES}

\section{A. Culture bactérienne}

La souche utilisée, Lactobacillus helveticus L89, provenait de l'Institut Rosell (Montréal). Cette bactérie homofermentaire produit de l'acide lactique D-L. Les cellules sont cultivées à $42^{\circ} \mathrm{C}$ dans un milieu de composition suivante : lactose $50 \mathrm{~g} / \mathrm{L}(\mathrm{BDH})$ extrait de levure $30 \mathrm{~g} / \mathrm{L}(\mathrm{BDH}), \mathrm{MgSO}_{4} 0,6 \mathrm{~g} / \mathrm{L}, \mathrm{FeSO}_{4} 0,03 \mathrm{~g} / \mathrm{L}, \mathrm{MnSO}_{4}$ $0,03 \mathrm{~g} / \mathrm{L}$, acétate de sodium $1,0 \mathrm{~g} / \mathrm{L}, \mathrm{K}_{2} \mathrm{HPO}_{4} 0,5 \mathrm{~g} / \mathrm{L}, \mathrm{KH}_{2} \mathrm{PO}_{4}$ 0,5 g/L. La souche est conservée à $4^{\circ} \mathrm{C}$ sur gélose MRS (Difco) (Man et al., 1960). Les cellules sont recueillies par centrifugation (9500 g, $10 \mathrm{~min}$ ) après $16 \mathrm{~h}$ de culture.

\section{B. Immobilisation des cellules}

La figure 1 représente le mode de fabrication du biocatalyseur. Après homogénéisation du culot bactérien avec la solution d'alginate de sodium, la suspension cellulaire était introduite dans une seringue stérile de $50 \mathrm{~mL}$. La répartition de la suspension en gouttes était assurée par un porte-filtre (Millipore, diamètre de $25 \mathrm{~mm}$, Bedford, MA) sur lequel 14 aiguilles hypodermiques (18 G 1/2) étaient fixées. $\mathrm{La}$ gélification des gouttes dans la solution de coagulation $\left(\mathrm{CaCl}_{2}\right.$ ou $\mathrm{BaCl}_{2}$ ) était immédiate. Les billes étaient ensuite laissées une heure sous une agitation douce afin de compléter l'échange ionique $\mathrm{Na}^{+}-\mathrm{Ca}^{++}$ou $\mathrm{Na}^{+}-\mathrm{Ba}^{++}$. Toutes ces opérations étaient réalisées aseptiquement. Les billes étaient alors lavées deux fois avec de l'eau distillée stérile afin d'éliminer l'excès de calcium. 

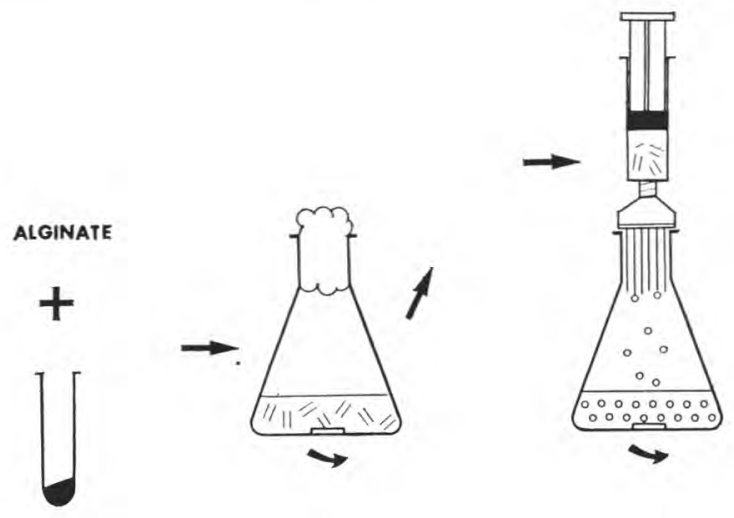

fig. 1

Schéma de fabrication du biocatalyseur

Première étape : Mélange du culot bactérien avec la solution stérile d'alginate.

Deuxième étape : Homogénéisation de la suspension.

Troisième étape : Extrusion des billes et gélification dans le bain de solution de $\mathrm{CaCl}_{2}$, sous agitation.

Diagram of biocatalyst production

First step : Mixing of cells with sterile alginate solution.

Second step : Homogenization.

Third step : The alginate suspension was extruded above a $\mathrm{CaCl}_{2}$ stirred coagulating bath.

\section{Conservation des billes}

Les cellules immobilisées étaient entreposées sur une période de 8 semaines à cinq températures différentes : température du laboratoire $\left(25^{\circ} \mathrm{C} \pm 2^{\circ} \mathrm{C}\right)$, réfrigération à $4^{\circ} \mathrm{C} \pm 2^{\circ} \mathrm{C}$, congélation à $-20^{\circ} \mathrm{C} \pm 3^{\circ} \mathrm{C}$ (vitesse de refroidissement de l'échantillon : $1^{\circ} \mathrm{C} /$ min), congélation à $-35^{\circ} \mathrm{C} \pm 3^{\circ} \mathrm{C}$ (même vitesse de refroidissement) et congélation dans l'azote liquide à $-196^{\circ} \mathrm{C}$. Les effets cryoprotecteurs de cinq milieux de conservation ont été testés : perméat préparé à partir de poudre de lactosérum doux réhydratée à $6,5 \%$, lait écrémé en poudre réhydraté à $9 \%$ et à $20 \%$, glycérol à $10 \%$ et d-sorbitol à $10 \%$.

\section{Préparation du perméat de lactosérum}

Le lactosérum était reconstitué à $6,5 \%$ de solides à partir de poudre de lactosérum provenant d'une fabrication de cheddar. Le perméat était obtenu par ultrafiltration (HF 15.43 - PM 50, Romicon HF 2SSS) et conservé à $-18^{\circ} \mathrm{C}$. Avant son utilisation, le perméat était stérilisé par filtration (Sartorius, $0,22 \mu$ ). 


\section{E. Activité fermentaire}

Cinquante millilitres de gel, sous forme de billes, étaient incubés pendant $4 \mathrm{~h}$ dans du perméat de lactosérum et l'acidité développée était mesurée.

\section{F. Procédés analytiques}

L'acide lactique était dosé suivant la méthode de Lawrence (1975). Les teneurs en sucre étaient mesurées par la méthode de Dubois et al. (1956).

\section{G. Relargage cellulaire}

$\mathrm{Au}$ cours de chaque expérimentation, des échantillons de perméat étaient prélevés, étalés sur géloses MRS et dénombrés.

\section{RESULTATS}

\section{A. Choix de l'alginate}

Après avoir testé plusieurs types d'alginate, de provenance et de marques différentes, notre choix s'est porté sur l'alginate à haute viscosité extrait de Macrocystis pyrifera (Sigma) (à $25^{\circ} \mathrm{C}$, une solution à $2 \%$ a une viscosité de 14000 centipoises). Celui-ci permettait d'obtenir des billes macroscopiquement homogènes sur une gamme de concentrations d'alginate de 0,5 à $2,5 \%(\mathrm{~m} / \mathrm{v})$.

\section{B. Détermination des concentrations optima d'alginate et de $\mathrm{CaCl}_{2}$ en vue de l'obtention des billes les plus résistantes à la dissolution (tab. 1)}

L'immobilisation de cellules dans un gel d'alginate est un procédé simple et peu coûteux. De plus, le gel présente une excellente résistance aux pressions hydrostatiques et à l'abrasion (Cheetham, 1979). Néanmoins, le gel peut être détruit si les ions calcium sont déplacés par un agent chélateur $\left(\mathrm{PO}_{4}^{-2}\right)$ ou par d'autres cations $\left(\mathrm{Mg}^{++}, \mathrm{K}^{+}\right)$. Nous avons donc cherché à obtenir un gel de grande résistance vis-à-vis de ce paramètre. Une solution à 2,5\% d'alginate donne des résultats très supérieurs aux autres concentrations. Malheureusement, la haute viscosité de cette solution implique de très fortes pressions pour la fabrication des billes. Cette concentration ne sera donc pas utilisée pour la suite de cette étude. De plus, les billes ainsi formées se présentaient sous la forme de gouttes et non sous la forme de billes parfaitement sphériques comme dans les autres concentrations. Les tailles des billes formées à l'aide du même appareillage et des différentes solutions sont 


\section{TABLEAU 1 - TABLE 1}

Temps (en minutes) de dissolution des billes dans un tampon tripolyphosphate $\mathrm{pH}$ 6,0

Duration (min) of beads dissolution in a tripolyphosphate buffer, $p H 6.0$

\begin{tabular}{lrrrrr}
\hline $\begin{array}{c}\text { Alginate } \\
(\%, \mathrm{M} / \mathrm{V})\end{array}$ & 0,5 & 1 & 1,5 & 2 & 2,5 \\
\hline $\mathrm{CaCl}_{2}$ & & & & & \\
$(\%, \mathrm{M} / \mathrm{V})$ & & & & & \\
0,5 & 50 & 90 & 120 & 125 & 155 \\
1 & 60 & 105 & 160 & 180 & 200 \\
2 & 105 & 180 & 960 & 1080 & $>1440$ \\
3 & 160 & 220 & 990 & 1080 & $>1440$ \\
\hline
\end{tabular}

N.B. : Les résultats sont la moyenne de 2 essais représentatifs.

\section{TABLEAU $2-T A B L E 2$}

Fabrication des billes en $\mathrm{CaCl}_{2}$ : diamètre moyen des billes en $\mathrm{mm}$ (sur 20 billes)

Production of beads with $\mathrm{CaCl}_{2}$ : mean diameter of beads in $\mathrm{mm}$ (for 20 beads)

\begin{tabular}{|c|c|c|c|c|c|}
\hline $\begin{array}{l}\text { Alginate } \\
\qquad(\%, M / V\end{array}$ & V) 0,5 & 1 & 1,5 & 2 & 2,5 \\
\hline \multicolumn{6}{|l|}{$\begin{array}{l}\mathrm{CaCl}_{2} \\
(\%, \mathrm{M} / \mathrm{V})\end{array}$} \\
\hline 0,5 & $2,0 \pm 0,1$ & $3,0 \pm 0,2$ & $3,0 \pm 0,2$ & $3,5 \pm 0,2$ & $3,5 \pm 0,2$ \\
\hline 1 & $2,0 \pm 0,1$ & $2,8 \pm 0,2$ & $3,0 \pm 0,2$ & $3,0 \pm 0,2$ & $3,5 \pm 0,2$ \\
\hline 2 & $1,8 \pm 0,2$ & $2,5 \pm 0,1$ & $3,0 \pm 0,1$ & $3,0 \pm 0,1$ & $3,0 \pm 0,2$ \\
\hline 3 & $2,0 \pm 0,1$ & $2,5 \pm 0,2$ & $2,5 \pm 0,2$ & $3,0 \pm 0,2$ & $3 ; 0 \pm 0,1$ \\
\hline
\end{tabular}

classées dans le tableau 2. Les billes de plus fort diamètre sont obtenues par de forts pourcentages d'alginate et de faibles concentrations en $\mathrm{CaCl}_{2}$.

\section{Polymérisation avec un cation divalent autre que $\mathrm{Ca}^{++}: \mathrm{Ba}^{++}$}

Les travaux de Haug et Smidsrød (1965), de Smidsr $\varnothing$ d et Haug (1965) et ceux de Paul et Vignais (1980) sur les agents inducteurs de la gélification ont montré que l'utilisation de chlorure de baryum 
permettait d'obtenir un gel plus dur et plus résistant que celui obtenu avec le chlorure de calcium. Les billes préparées à l'aide de $\mathrm{BaCl}_{2}(50 \mathrm{mM}, \mathrm{pH} 6,0)$ étaient de taille inférieure à celles obtenues en $\mathrm{CaCl}_{2}$ dans les mêmes conditions. Leur masse était également plus faible : $5 \mathrm{~g}$ gel alginate- $\mathrm{CaCl}_{2}=250 \pm 20$ billes tandis que $5 \mathrm{~g}$ gel alginate- $\mathrm{BaCl}_{2}=520 \pm 30$ billes. Malheureusement, la toxicité du chlorure de baryum (Paul et Vignais, 1980) pourrait être un obstacle à l'utilisation des billes ainsi fabriquées (par un éventuel relargage du baryum dans le produit) et par là même annihiler les avantages liés à l'utilisation de produits de catégorie alimentaire.

\section{Efficacité de l'immobilisation}

La détermination du nombre de cellules viables immobilisées dans les billes nécessite la dissolution préalable du gel. Cette déstabilisation du gel ne doit pas altérer la viabilité des cellules. Le tableau 3 représente les temps nécessaires à la dissolution d'un ensemble de 20 à 25 billes dans $10 \mathrm{ml}$ de solution, avec agitation. Le citrate de sodium et le tampon tri-polyphosphate s'avèrent les deux composés les plus efficaces pour la dissolution des billes. On remarque l'effet marqué de la solution de lactate de sodium à $3 \%$ et $4 \%$. Cette solubilité du gel dans le lactate (produit de la réaction considérée dans cette étude) nous conduira à essayer de rendre le polymère plus résistant par un taitement chimique approprié.

\section{TABLEAU $3-T A B L E 3$}

Temps de dissolution de billes ( $1 \%$ alginate, $50 \mathrm{mM} \mathrm{CaCl}$ ) (moyenne de 2 essais représentatifs)

Duration of beads dissolution (1\% alginate, $50 \mathrm{mM} \mathrm{CaCl}$ ) (average of two representative trials)

\begin{tabular}{|c|c|c|}
\hline Solutions & $\begin{array}{c}\text { Temps } \\
\text { de } \begin{array}{c}\text { dissolution } \\
(\mathrm{mn})\end{array}\end{array}$ & $\begin{array}{l}\text { Numération } \\
\text { sur MRS } \\
\text { (cellules/bille) }\end{array}$ \\
\hline $\begin{array}{l}\text { Lactate de } \mathrm{Na}^{+} \mathrm{pH} 6,0 \\
1 \% \\
2 \% \\
3 \% \\
4 \%\end{array}$ & $\begin{array}{r}1440 \\
960 \\
98 \\
62\end{array}$ & $\begin{array}{l}10^{6} \text { à } 5 \cdot 10^{6} \\
10^{6} \text { à } 5 \cdot 10^{6} \\
10^{6} \text { à } 8 \cdot 10^{7} \\
10^{6} \text { à } 2 \cdot 10^{7}\end{array}$ \\
\hline Phosphate de $\mathrm{Na}^{+} 100 \mathrm{mM}$ pH 6,0 & 1440 & $10^{\circ}$ à $6 \cdot 10^{6}$ \\
\hline Citrate de $\mathrm{Na}^{+} 1 \% \mathrm{pH} 6,0$ & 44 & $10^{6}$ à $6 \cdot 10^{6}$ \\
\hline Tripolyphosphate saturé $\mathrm{pH} 6,0$ & 43 & $2 \cdot 10^{6}$ \\
\hline
\end{tabular}


TABLEAU $4-T A B L E 4$

Comparaison de conservation des micro-organismes immobilisés

Comparison of conservation for immobilized microorganisms

\begin{tabular}{|c|c|c|c|c|c|c|}
\hline Microorganismes & Type d'immobilisation & $\begin{array}{c}\text { Temps de } \\
\text { conservation }\end{array}$ & Traitement & $\begin{array}{c}\text { Perte } \\
\text { d'activité }\end{array}$ & $\begin{array}{l}\text { Solution de } \\
\text { conservation }\end{array}$ & Réference \\
\hline Lactobacillus delbruecki i & Alginate de calcium & 157 jours & $7^{\circ} \mathrm{C}$ & $10 \%$ & $\begin{array}{c}4,8 \% \\
\text { glucose }\end{array}$ & Stenroos et al. (1982a) \\
\hline Lactobacillus delbruecki i & Alginate de calcium & 150 jours & $4^{\circ} \mathrm{C}$ & $10 \%$ & - & Stenroos et al. (1982b) \\
\hline Lactobacillus delhrueckli & Alginate de calcium & - & $4^{\circ} \mathrm{C}$ & $0 \%$ & - & $\begin{array}{l}\text { Linko (1981) } \\
\text { Linko et Linko (1981) }\end{array}$ \\
\hline Lactobacillus helveticus & Alginate de calcium & 56 jours & $\begin{array}{l}-196^{\circ} \mathrm{C} \\
-\quad 35^{\circ} \mathrm{C} \\
-20^{\circ} \mathrm{C} \\
+\quad 4{ }^{\circ} \mathrm{C} \\
+\quad 25^{\circ} \mathrm{C}\end{array}$ & $\begin{array}{c}0 \% \\
0 \% \\
15 \% \\
- \\
25 \%\end{array}$ & $\begin{array}{l}\text { Perméat de } \\
\text { lactoserrum }\end{array}$ & Cet article \\
\hline Photobacterium phosphoreum & Alginate de calcium & 32 jours & $-15^{\circ} \mathrm{C}$ & $0 \%$ & $\begin{array}{l}10 \% \text { glycérol } \\
10 \% \text { sorbitol } \\
10 \% \text { thréonine }\end{array}$ & Makiguchi et al. (1980) \\
\hline$\frac{\text { Brevibacterium }}{\text { ammoniagenes }}$ & Polyacrylamide & 45 jours & $0-4^{\circ} \mathrm{C}$ & $0 \%$ & $\begin{array}{l}\text { Tampon phosphate } \\
\text { de potassium } \\
0,01 \mathrm{M}, \mathrm{pH} 7,5\end{array}$ & Shimizu et al. (1975, 1979) \\
\hline Arthrobacter simplex & ENT $4000 / E N T 2000$ & 30 jours & $\begin{array}{r}20^{\circ} \mathrm{C} \\
4^{\circ} \mathrm{C}\end{array}$ & $\begin{array}{r}0 \% \\
50 \%\end{array}$ & $\begin{array}{l}\text { Tampon phosphate } \\
\text { de potassium } \\
20 \mathrm{~mm}, \mathrm{pH} 7.0\end{array}$ & Sonomoto et al. (1979) \\
\hline Clostridium butyricum & Polyacrylamide & 30 jours & $5^{\circ} \mathrm{C}$ & $0 \%$ & $\begin{array}{l}\text { Tampon } 0,1 \mathrm{M} \\
\text { phosphate, } \\
\text { pH } 7,7\end{array}$ & Karube et al. (1976) \\
\hline Curvularia lunata & Polyacrylamide & 24 heures & $2^{\circ} \mathrm{C}$ & $50 \%$ & - & Ohlson et al. (1980) \\
\hline Curvularia lunata & Polyacrylamide & 7 jours & $4^{\circ} \mathrm{C}$ & $50 \%$ & - & Mosbach et Larsson (1970) \\
\hline
\end{tabular}




\section{E. Viabilité des cellules de Lactobacillus helveticus dans les solutions de dissolution du gel}

Devant les temps de dissolution des billes, il s'avère nécessaire d'évaluer le pourcentage de survie des cellules au cours de l'opération. Des suspensions cellulaires sont incubées à $25^{\circ} \mathrm{C}$ dans $10 \mathrm{~mL}$ de tampon citrate de sodium ( $\mathrm{pH}$ 6,0) ou de tampon tri-polyphosphate $(\mathrm{pH} 6,0)$. Les suivis de viabilité sont effectués par dénombrement direct, après passage au vortex, sur géloses MRS (fig. 2A et 2B). On observe une chute très nette de la viabilité en tampon tri-polyphosphate tandis que celle-ci n'est que peu altérée par un séjour des cellules en tampon citrate de sodium. Nous utiliserons ce dernier dans la suite de cette étude.
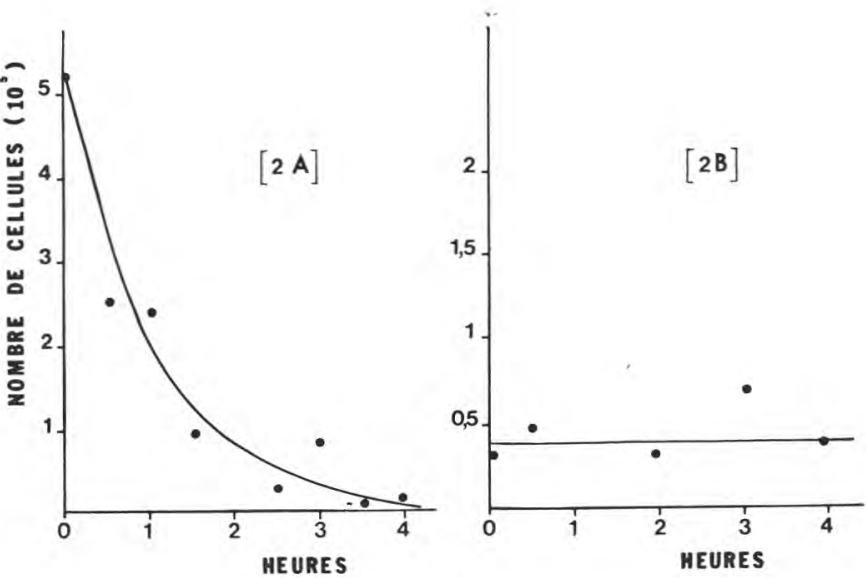

fig. 2

Viabilité des cellules de L. helveticus après incubation dans une solution saturée de tripolyphosphate de sodium à $\mathrm{pH} 7,0(2 \mathrm{~A})$ ou dans une solution de citrate de sodium (1\% M/V) pH 6,0 (2B) (Dénombrements sur géloses MRS).

Viability of L. helveticus cells after incubation in a saturated solution of sodium tripolyphosphate, $p H$ 7.p $(2 A)$ or in a solution of sodium citrate $(1 \% \mathrm{~W} / \mathrm{V})$ pH 6.0 (2B) (Plate counts on MRS agar).

\section{F. Traitement de stabilisation du gel}

L'un des problèmes majeurs des cellules entières vivantes immobilisées est sans doute le relargage des cellules dans le milieu. Pour remédier à cet inconvénient, divers traitements chimiques ont été utilisés : polylysine (Lim et Sun, 1980), glutaraldéhyde, gélatine + glutaraldéhyde, hexaméthylènediamine (Takata et al., 1977), polyé- 
thylèneimine (PEI) Birnbaum et al., 1982 ; Veliky et Williams, 1981). Les billes contenant les cellules de Lactobacillus helveticus ont été soumises à divers traitements de stabilisation. Le PEI provoque une sévère contraction des billes dont le diamètre devient inférieur au millimètre. Cette forte diminution de taille s'accentue si les billes sont ensuite placées dans un milieu nutritif. Cet effet est toutefois moins prononcé lorsque plusieurs lavages précèdent cette incubation. Le relargage des cellules demeure néanmoins important dans les deux cas. Le glutaraldéhyde, employé à la concentration classiquement utilisée pour cet effet $(200 \mathrm{mM})$ (Takata et al., 1977) provoque la mort des cellules. D'autres essais ont été réalisés à l'aide de tannins. Si la viabilité et l'activité des cellules ne sont pas altérées par ce traitement, les temps de dissolution des billes ne sont pas significativement augmentés. Nous avons donc décidé de ne pas soumettre les futurs catalyseurs à ces traitements.

\section{G. Effets des différentes températures de conservation sur l'activité des cellules immobilisées}

La figure 3 montre l'activité fermentaire de $50 \mathrm{~mL}$ de billes d'alginate de calcium contenant des cellules de Lactobacillus helveticus entreposées à différentes températures. Les échantillons conservés dans le lait ont une activité supérieure à celle des billes entreposées dans le perméat de lactosérum. Aucune perte d'activité n'a été enregistrée après congélation sauf pour les échantillons conservés à $-20^{\circ} \mathrm{C}$ dans le perméat. Ceux-ci présentent une perte d'activité de $10 \%$ après 8 semaines de congélation, ce qui corrobore les résultats de Larsson et Mosbach (1976). Cette perte d'activité s'accompagne d'une baisse du nombre de cellules. La réfrigération à $4^{\circ} \mathrm{C}$ permet une bonne stabilité de l'activité bactérienne : $100 \%$ après 8 semaines. Aucun résultat n'est enregistré pour les échantillons conservés dans le perméat de lactosérum pour cette série, mais d'autres essais parallèles ont montré une bonne stabilité à $4^{\circ} \mathrm{C}$. A $25^{\circ} \mathrm{C}$, les lactobacilles possèdent une activité acidifiante suffísamment importante pour abaisser le $\mathrm{pH}$ du milieu à un point toxique, voire léthal (résultats non montrés).

\section{H. Effets des différentes températures de conservation sur le nombre de cellules viables dans les billes}

Le nombre de cellules vivantes demeure stable au cours des 8 semaines de congélation à $-196^{\circ} \mathrm{C},-35^{\circ} \mathrm{C},-20^{\circ} \mathrm{C}$ et à $4^{\circ} \mathrm{C}$. Le type de milieu de conservation (lait ou perméat de lactosérum) ne semble pas exercer une influence sur la survie des lactobacilles. La conservation à $25^{\circ} \mathrm{C}$ provoque une chute brutale du nombre de cellules viables de par l'acidification du milieu (résultats non montrés). 


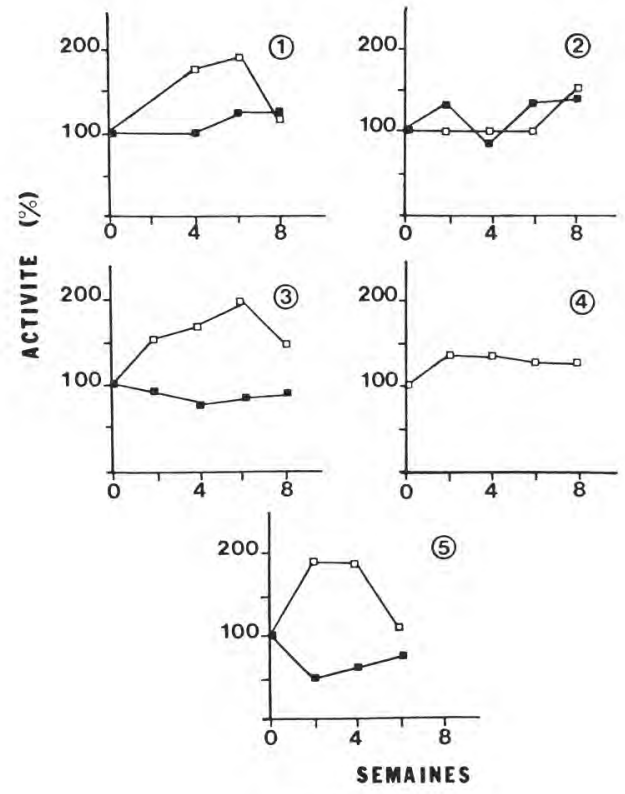

fig. 3

Evolution de l'activité fermentaire de $50 \mathrm{~mL}$ de billes d'alginate contenant les cellules de L. helveticus, dans du lait $(\square)$ et du perméat ( $\square$ ), au cours de 8 semaines d'entreposage à $-196^{\circ} \mathrm{C}(1),-35^{\circ} \mathrm{C}(2),-20^{\circ} \mathrm{C}$ (3), $4^{\circ} \mathrm{C}$ (4) et $25^{\circ} \mathrm{C}(5)$.

Evolution of the fermentation activity of $50 \mathrm{~mL}$ alginate beads containing $\mathrm{L}$. helveticus cells, in milk ( $\square$ ) and permeate (1)during an 8-week storage period at $-196^{\circ} \mathrm{C}(1),-35^{\circ} \mathrm{C}(2),-20^{\circ} \mathrm{C}(3), 4^{\circ} \mathrm{C}$ (4) and $25^{\circ} \mathrm{C}$ (5).

\section{Effets des différentes températures de conservation sur l'intégrité des billes et le relargage cellulaire}

Il est important de conserver la forme des billes d'alginate pour deux raisons principales. La sphère est la forme géométrique permettant le maximum d'échanges. De plus, les billes permettent d'obtenir un écoulement régulier de la solution à fermenter dans le type de bioréacteur que nous utilisons (« packed bed reactor »). A $25^{\circ} \mathrm{C}$ et à $4^{\circ} \mathrm{C}$, les billes gardent leur intégrité (examen macroscopique) et le relargage des cellules est peu important (après 8 semaines de conservation, $8 \mathrm{~h}$ d'incubation donnent un relargage inférieur à 20000 cellules $/ \mathrm{mL}$ ). Par contre, les billes congelées à $-20^{\circ} \mathrm{C}$ et à $-35^{\circ} \mathrm{C}$ ont une apparence flétrie, poreuse, translucide, qui s'accompagne d'un important relargage cellulaire (supérieur à 300000 cellules $/ \mathrm{mL}$, pour les mêmes conditions que précédemment). Les billes conservées dans l'azote liquide $\left(-196^{\circ} \mathrm{C}\right)$ 
ont gardé une forme sphérique parfaite et présentent un relargage cellulaire faible.

\section{J. Effets de quelques solutions cryoprotectrices sur la conservation de l'intégrité des billes}

Des substances reconnues comme barrières efficaces contre les effets négatifs entraînés par la congélation sur les cellules ont été ajoutées à la préparation de conservation du gel : sorbitol $10 \%$, glycérol $10 \%$, poudre de lait écrémé réhydraté à $20 \%(\mathrm{~m} / \mathrm{v})$. Ces solutions, contenant $50 \mathrm{~mL}$ de billes, ont été entreposées à $-20^{\circ} \mathrm{C}$ pendant 2 semaines. Des billes macroscopiquement déformées ont été obtenues dans tous les cas. Par ailleurs, on n'observait pas de variation de l'activité acidifiante des cellules ainsi conservées par comparaison avec des cellules conservées dans du perméat de lactosérum sans ajout.

\section{DISCUSSION}

L'immobilisation de cellules dans des billes d'alginate de calcium à $2 \%(\mathrm{~m} / \mathrm{v})$ d'alginate permet d'obtenir un biocatalyseur de manipulation aisée. Le procédé est assez doux pour permettre une bonne viabilité des cellules dans la bille après la gélification, phénomène indispensable dans le cas d'une transformation multi-enzymatique (Slowinski et Charm, 1973). Les traitements de stabilisation du gel n'ont pas apporté un bénéfice suffisant aux propriétés rhéologiques des billes pour être maintenus. De plus, l'utilisation du perméat de lactosérum comme substrat nous permet d'éviter un relargage important de calcium, néfaste à la structure du gel. En effet, l'ion calcium est présent en quantités importantes dans le perméat et semble contrebalancer efficacement l'effet chélateur de l'acide lactique produit. Des essais complémentaires mettant en jeu des concentrations plus importantes d'acide lactique que celles utilisées dans ces travaux sont en cours pour vérifier ce point. L'utilisation du citrate de sodium comme déstabilisateur du gel répond aux critères de viabilité indispensables. Le temps de dissolution pourrait probablement être diminué par un broyage mécanique des billes afin d'augmenter les surfaces de contact biocatalyseur-citrate. Les cellules de Lactobacillus helveticus immobilisées peuvent être conservées de façon satisfaisante pendant 2 mois sans perte d'activité et sans perte de l'intégrité des billes à $-196^{\circ} \mathrm{C}$ et à $+4^{\circ} \mathrm{C}$. Une conservation à $+25^{\circ} \mathrm{C}$ nécessiterait un milieu plus tamponné. La congélation lente semble induire une cristallisation dans les billes qui perturbe définitivement l'intégrité du gel. D'autres essais pourraient être envisagés sur le séchage partiel des billes d'alginate, 
sous atmosphère contrôlée, comme le préconisent Klein et Wagner (1978). Des essais de lyophilisation ont été entrepris. La réhydratation du matériel rétablit l'intégrité structurale du biocatalyseur. Néanmoins, la lyophilisation de grandes quantités de matériel destiné à être incubé dans un milieu riche est un procédé coûteux qui, de plus, présente des problèmes d'aseptie. Larsson et Mosbach (1976) notaient une baisse de $60 \%$ de l'activité immédiatement après la lyophilisation de cellules immobilisées d'Arthrobacter simplex dans un gel de polyacrylamide. Le tableau 4 permet une comparaison entre les efficacités de diverses méthodes de conservation. Les résultats des échantillons congelés se situant très au-dessus de $100 \%$ d'activité ne peuvent pas être considérés comme de simples variations d'échantillonnage (fig. 3). La congélation peut provoquer certains changements de la membrane cellulaire qui entraînent parfois une plus grande perméabilité aux nutriments ou (et) aux produits du métabolisme. De plus, un double rinçage à l'eau distillée des billes conservées dans le lait ne suffit peut-être pas à éliminer toutes les protéines présentes, protéines qui sont absentes du perméat dans lequel elles sont ensuite placées pour le test d'activité. Ces protéines pourraient avoir des effets positifs au niveau de l'activité cellulaire en fournissant une source supplémentaire d'azote. De façon globale, l'utilisation des sucres par les échantillons décroît au cours de la conservation. On observe une augmentation du temps de dissolution des billes au cours de la conservation. La conservation du gel altérant parfois leur homogénéité, cette augmentation du temps de dissolution peut surprendre. Les résultats des travaux relatifs aux optima de production d'acide lactique par les cellules immobilisées de Lactobacillus helveticus L89 ( $\mathrm{pH}$, température, stabilité thermique, etc.) sont publiés ailleurs. La mise au point, à l'échelle du laboratoire, d'une fermentation en continu du perméat, à l'aide d'une colonne contenant les billes d'alginate, est en cours. Nous examinons également la possibilité de découpler l'activité acidifiante des bactéries de la croissance Iorsque celle-ci est arrivée à un optimum. Les problèmes liés à l'utilisation des bactéries lactiques (bactériophages) en présence de calcium représente également un des points que nous examinons.

\section{REFERENCES BIBLIOGRAPHIQUES}

Birnbaum S., Pendieton R., Larsson P.O., Mosbach K., 1982. Covalent stabilization of alginate gel for the entrapment of living whole cells. Biotechnol. Lett., 3, 393-400.

CHEETHAM P.S.J., 1979. Physical studies on the mechanical stability of columns of calcium alginate gel pellets containing entrapped microbial cells. Enzyme Microb. Technol., 1, 183-188.

Chibata I., Tosa T., 1981. Use of immobilized cells. Ann. Rev. Biophys. Bioeng., $10,197-216$. 
Dubois M., Gilles K.A., Hamilton J.K., Rebers P.A., Smith F., 1956. Colorimetric method for the determination of sugars and related substances. Anal. Chem., 28, 350-356.

Durand G., Navarro J.M., 1978. Immobilized microbial cells. Proc. Biochem., 14-23.

Fukur S., TANaKa A., 1982. Immobilized microbial cells. Ann. Rev. Microbiol., 36, $145-172$.

HaUG A., SMIDSR $\phi$ D O., 1965. The effect of divalent metals on the properties of alginate solutions. II. Comparison of different metal ions. Acta Chem. Scand., $19,341-351$.

Karube I., Matsunaga T., Tsuru S., Suzuki S., 1976. Continuous hydrogen production by immobilized whole cells of Clostridium butyricum. Biochim. Biophys. Acta, 444, 338-343.

KLeIN J., Wagner F., 1978. Immobilized whole cells. In Dechema Monographien. Proc. First European Congress on Biotechnology, 82, Verlag Chemie Weinheim, New York, 142-164.

LARSSON P.O., MOSBACH K., 1976. Immobilization of steroid-transforming microorganisms in polyacrylamide. Methods in Enzymology, ed. Mosbach K., Acad. Press, New York, XLIV, 183-190.

Lawrence A.J., 1975. Determination of lactic acid in cream. Australian J. Dairy Technol., 30, 14-15.

LiM F., Sun A.M., 1980. Microencapsulated islets as bioartificial endocrine pancreas. Science, 210, 908-910.

Linko P., 1981. Immobilized live cells. Advances in Biotechnology, 1, 711-716. Ed. by M. Moo Young, C.W. Robinson and C.. Vezzina, Pergamon Press, New York.

Linko P., Linko Y.Y., 1981. Immobilized microbial cells for ethanol and other applications. Condensed preprint of paper $69 \mathrm{a}$ presented at 74 th Annual AICHE Meeting, New Orleans, Louisiana.

Makiguchi N., ARita M., Asar Y., 1980. Optimal conditions for frozen storage of immobilized luminous bacteria. J. Ferment. Technol., 58, 333-337.

Man J.C., Rogosa M., ShaRpe M.E., 1960. A medium for the cultivation of Lactobacilli. J. Appl. Bacteriol., 23, 130-135.

Mosbach K., LaRsson P.O., 1970. Preparation and application of polymer-entrapped enzymes and microorganisms in microbial transformation processes with special reference to steroid $11 \beta$-hydroxylation and $\Delta^{1}$ dehydrogenation. Biotechnol Bioeng., 12, 19-27.

Ohlson S., Flygare S., LaRsSon P.O., Mosbach K., 1980. Steroid hydroxylation using immobilized spores of Curvularia lunata germinated in situ. Europ. J. Appl. Microbiol. Biotechnol., 10, 1-9.

PaUl F., Vignais P.M., 1980. Photophosphorylation in bacterial chromatophores entrapped in alginate gel : improvement of the physical and biochemical properties of gel beads with barium as gel inducing agent. Enzyme Microb. Technol., 2, 281-287.

Shimizu S., Morioka H., Tani Y., Ogaka K. 1975. Synthesis of coenzyme A by immobilized microbial cells. J. Ferment. Technol., 53, 77-83.

Shimizu S., TANi Y., Yamada H., 1979. Synthesis of coenzyme A by immobilized microbial cells. In : Immobilized Microbial Cells, ASC Symposium series 106, 87-100.

SlowinsKi W., ChaRM S.E., 1973. Glutamic acid production with gel-entrapped Corynebacterium glutamicum. Biotech. Bioeng., 15, 973-979. 
SmidsRøi O., HaUg A., 1965. The effect of divalent metals on the properties of alginate solution. I. Calcium ions. Acta Chem. Scand., 19, 329-340.

Sonomoto K., Tanaka A., Omata T., Yamane T., Fukui S., 1979. Application of photo-crosslinkable resin prepolymers to entrap microbial cells. Effects of increased cell-entrapping gel hydrophobicity on the hydrocortisone $\Delta^{1}$-dehydrogenation. Europ. J. Appl. Microbiol. Biotechnol., 6, 325-334.

StenRoos S.L., Linko Y.Y, Linko P., 1982 a. Production of lactic acid with immobilized Lactobacillus delbrueckii. Biotechnol. Lett., 4, 159-164.

Stenroos S.L., Linko Y.Y., Linko P., HaRJu M., Hetkonen M., 1982 b. Lactic acid fermentation with immobilized Lactobacillus sp. Enzyme Eng., 6, 299-301.

Takata I., Tosa T., Chibata I., 1977. Screening of matrix suitable for immobilization of microbial cells. J. Solid Phase Biochem., 2, 225-236.

Veliky I.A., Williams R.E., 1981. The production of ethanol by Saccharomyces cerevisiae immobilized in polycation-stabilized calcium alginate gels. Biotechnol. Lett., 3, 275-280. 